Tropical Journal of Pharmaceutical Research November 2015; 14 (11): 2131-2134

ISSN: $1596-5996$ (print); 1596-9827 (electronic)

(c) Pharmacotherapy Group, Faculty of Pharmacy, University of Benin, Benin City, 300001 Nigeria.

All rights reserved.

Available online at http://www.tjpr.org

Case Report

http://dx.doi.org/10.4314/tjpr.v14i11.26

\title{
A Report on Antibiotic Management of Neonatal Sepsis Caused by Enterobacter spp
}

\section{Ricardo Alexandre Spironello', Clarissa Botura-Amado', Thiely Karine Balensiefer ${ }^{1}$, Maria Dalva de Barros Carvalho ${ }^{2}$, Darci Aparecida Martins Corrêa $^{3}$ and Roberto Kenji Nakamura Cuman ${ }^{1 *}$}

${ }^{1}$ Department of Pharmacology and Therapeutics, ${ }^{2}$ Department of Medicine, ${ }^{3}$ Department of Nursing, State University of Maringá, Paraná, Brazil

*For correspondence: Email: rkncuman1@gmail.com

Received: 7 July 2015

Revised accepted: 8 October 2015

\begin{abstract}
This is a report on a case of neonatal sepsis and clinical management with multiple antibiotic therapy in a neonatal intensive care unit (NICU) in Brazil. A preterm baby boy was born by caesarean section at 34 weeks and two days of gestation from an oligodramnious pregnancy with intrauterine growth restriction. After respiratory failure detection, the baby was intubated and placed on mechanical ventilation for respiratory failure and was shifted to NICU. Ampicillin and gentamicin were instituted empirically. Enterobacter spp-induced severe sepsis was diagnosed. Included in the antibiotic therapy were oxacycline, amikacin, cefepime, tazocin, meropen and teicoplanin. After sixty days in NICU, clinical and laboratory parameters were normalized and the baby recovered. The empirical long-term antibiotic treatment and the use of broad spectrum antibiotics, as observed in the present case, should be carefully considered in newborns admitted to NICU.
\end{abstract}

Keywords: Sepsis, Newborn, Respiratory failure, Multiple antibiotic therapy

Tropical Journal of Pharmaceutical Research is indexed by Science Citation Index (SciSearch), Scopus, International Pharmaceutical Abstract, Chemical Abstracts, Embase, Index Copernicus, EBSCO, African Index Medicus, JournalSeek, Journal Citation Reports/Science Edition, Directory of Open Access Journals (DOAJ), African Journal Online, Bioline International, Open-J-Gate and Pharmacy Abstracts

\section{INTRODUCTION}

Neonatal bacterial sepsis, classified as early or late onset, is a major cause of morbidity and mortality, particularly for premature neonates, being an important public health concerns worldwide [1]. Very low birth weight infants (VLBW), weighing between 500 and $1500 \mathrm{~g}$ are known to have a high risk of acquiring sepsis [2], despite many advances in obstetrical and neonatal care [2]. The incidence of neonatal sepsis in developed countries ranged from 10-25 $\%$ of all infants and reached up to $50 \%$ in VLBW infants [1]. Neonatal infections are considered infections derived either intrapartum from the mother's flora, or acquired in the hospital
[3].Early onset neonatal sepsis (EOS) occurs in the first three days of life, which is typically caused by organisms transmitted vertically from the mother to the infant before or at the time of birth [4]; while late onset neonatal sepsis (LOS), an infection occurring after day 3 of life, is more likely to be caused by pathogens acquired during the course of hospital care. Besides higher risk of mortality caused by neonatal sepsis, survivors of neonatal sepsis may develop severe morbidity secondary to concomitant meningitis or from hypoxemia resulting from persistent pulmonary hypertension, septic shock, and respiratory failure [5]. Early onset neonatal sepsis (EOS) is reported to be caused primarily by Streptococcus agalactiae and Escherichia coli in high income 
countries [6]. In developing countries, bacteria like Klebsiella spp., Escherichia coli, and Staphylococcus aureus are reported to be the main cause reason of neonatal sepsis within the first week of life [7]. The spectrum of bacteria seen in early onset newborn sepsis also depends on the gestational week [8]. In mothers who received intrapartum or prepartum antibiotic treatment, there is likelihood to encounter infections with Enterobacter or Klebsiella spp. [5]. In recent years, the incidence of infections with Gram-negative rods such as Escherichia coli, Enterobacter spp, and Klebsiella spp seemed to increase in many neonatal intensive care unit (NICU) worldwide [5].

Enterobacteriaceae was noted to pose an increasing problem in the recent years, causing severe nosocomial infections. Besides rapid diagnosis, the immediate initiation of treatment is of great importance for a favorable treatment outcome. Suspected infections are quite frequent in premature newborns and empirical antibiotic therapy is an emergency. If treatment is delayed or ineffective, neonatal sepsis can be rapidly fatal, making optimal use of antibiotics is most essential. Empiric antibiotic therapy in newborns is based on combinations of cephalosporin, ampicillin and gentamicin [9,10]. A prolonged empiric antibiotic therapy after birth, however, was shown to be problematic in pre-term infants. Thus, either toxic or subtherapeutic treatment courses may exist in the neonatal clinical care, promoting adverse reactions and antibiotic resistance which obviously impacts clinical outcomes.

We report a clinical course of antibiotic therapy occurred in a NICU from Brazil.

\section{CASE REPORT}

A preterm baby boy with birth weight of $2.14 \mathrm{~kg}$ was by caesarean delivery at 34 weeks and two days of gestation at a public hospital, in a city of Paraná State, Brazil, in April 2014. The oligodramnious pregnancy of twins was diagnosed with intrauterine growth restriction and presence of grade II placenta. One of the twins died after caesarean. The surviving infant was well at birth, with Apgar scores of 8 and 10, at 1 and $5 \mathrm{~min}$, respectively. The temperature was $36.50{ }^{\circ} \mathrm{C}$, heart rate $134-138 / \mathrm{min}$, respiratory rate $36-46 / \mathrm{min}$, perfusion < $3 \mathrm{~s}$. Since the respiratory course deteriorated with worsening saturation, the baby was intubated and placed on mechanical ventilation for respiratory failure. On the second day of birth, the infant presented with jaundice and respiratory failure and was shifted to NICU. The symptoms were suggestive respiratory distress syndrome (RDS) or pulmonary infection. The hematological parameters and blood culture were investigated. Ampicillin (200 mg,i.v.,12 h) and gentamicin (9 $\mathrm{mg}$, i.v., $36 \mathrm{~h}$ ) were instituted empirically. Cardiovascular drugs, such as dobutamine (6 $\mathrm{mg} / \mathrm{kg} / \mathrm{min}$ ), and opioid (fentanyl, $2 \mathrm{mg} / \mathrm{kg} / \mathrm{min}$ ) were administered and secondary hypertensive state was observed. At 6th day in the NICU, hypoglycemia was observed and thrombocytopenia was not elicited $(136,000 \times$ $10^{9}$ platelets/L). Dexamethasone $(0.3 \mathrm{mg}$, i.v., $6 \mathrm{~h})$ was also introduced to avoid broncopulmonary dysplasia. At 9th day, the results of blood culture were not yet available, bradycardia was detected and antibiotic therapy was changed to oxacilin (50 mg, i.v., $12 \mathrm{~h}$ ) and amikacin (28 mg, i.v., 24 h), suggesting low efficacy of gentamicin and ampicillin combination and urine culture was negative. In the following days, bradycardia was restored by dobutamine administration. The antibiotic cefepime (93 mg, i.v., $12 \mathrm{~h}$ ) was also included in the treatment. The investigations revealed leukocytosis, thrombocytopenia $(26,000$ $\times 10^{9}$ platelets/L) and C-reactive protein. Platelet transfusion was required. On 15th day blood culture yielded, Enterobacter spp, confirming the diagnosis of severe sepsis. Tazocin $(180 \mathrm{mg}, 8$ h) was immediately administrated.

Two days after, pneumoperitoneum was observed and thrombocytopenia was continuous $\left(32,000 \times 10^{9}\right.$ platelets/L). The appendix was determined as perfored and obstructed and appendectomy was performed. Severe alterations in haemogram and C-reactive protein were observed and in the antibiotic therapy were included meropenem (40 mg, i.v., $8 \mathrm{~h}$ ) and teicocloplanin (20 mg; i.v., $12 \mathrm{~h}$ ). As the clinical state worsened, vancomycine (30 mg, i.v., $8 \mathrm{~h}$ ) and mycamine (20 mg, i.v., 24 h) were introduced, but it caused seizure state and ascite formation. Furosemide (20 mg, i.v., $12 \mathrm{~h}$ ) was recommended, since oliguria and hypervolemia were presented. At this stage platelet level was $8.000 \times 10^{9}$ platelets/L, with continuous antibiotic therapy. Since many drugs were used in this clinical case, drug-drug interaction was possible. However, using the Naranjo Adverse Drug Reaction Probability Scale, it was observed that a "possible adverse drug reaction" (score 4), related to a continuous thrombocytopenia.From 30th and following days, clinical and laboratory parameters were normalized. One month after discharge from NICU, the infant was in good general condition. 
Data collection was done retrospectively on the basis of medical records. Newborn sepsis was defined as acquiring a sepsis within 30 days after birth. Early onset sepsis was defined as having a positive blood culture within $72 \mathrm{~h}$ and late onset sepsis after $72 \mathrm{~h}$ of delivery. Additionally, diagnosis of sepsis was based on having at least two clinical signs of sepsis (core temperature $>38.5^{\circ} \mathrm{C}$, tachycardia/bradycardia or tachypnea) and one positive laboratory parameter linked to sepsis, i.e., white blood cell count and a Creactive protein (CRP).

\section{Ethical approval}

This report was approved (approval no 919.213COPEP/UEM) by Ethics Committee of State University of Maringá, Brazil.

\section{DISCUSSION}

Early onset neonatal sepsis was reported within the first week of life. Many factors are involved in the onset of sepsis, including: gestational age, caesarean or spontaneous vertex delivery, Apgar score and infant weight. Gram negative bacteria such as Enterobacter spp played an important role in neonatal sepsis in NICUs [5-7] predominantly in premature newborns with VLBW (below 2,500 g) and in one-third of cases, it was related to surgical interventions or previous antibiotic therapy during pregnancy period [12].

As microbiologically evaluated infection is rare in neonates, treatment has to be started as early as possible and merely upon clinical suspicion. Indeed, faster and specific tests are necessary to avoid the inappropriate use of antibiotics, since it has been reported multiresistant bacterial strain. Failure to recognize that resistant strains may result in ineffective antibiotic therapy with its attendant morbidity and mortality.

The initial choice of antibiotic therapy will depend on the clinical context and local bacterial epidemiology. In all cases, newborns who acquired an infection with Enterobacteriaceaeare empirically treated with ampicillin, gentamicin and/or cephalosporin before the onset of the sepsis. In this context, prolonged ( $>5$ days) empirical treatment/prophylaxis with broad spectrum antibiotics was shown to be associated with a higher risk of late onset sepsis (LOS), necrotizing enterocolitis and mortality in preterm infants $[11,12]$. In this study, it was observed that the mean duration of empirical antibiotic treatment was 7 days. This is noteworthy in view of the high resistance and mortality rates often seen in newborns with LOS due to failure of empirical treatment. We can only speculate that some resistance was caused by strain selection due to long-term initial antibiotic therapy or passed on to the newborns via their mothers around birth. The present case is early onset sepsis and the baby could have acquired from the mother. At the moment it is not possible to give a concluding statement on this question.

The possibility of a nosocomial infections outbreak of multi-resistant strains is argued against by the fact that there was no timely gathering of cases. Here, we are furthermore limited by the fact that we are not able to provide data about various bacterial strains involved in this case. Indeed, the treatment of neonatal sepsis is a challenge. The neonate had immunosuppression or debilitation because of prematurity, respiratory tract instrumentation, NICU stay and use of broad spectrum antibiotics. The treatment needs to be rapid, appropriate for the pathogen and safety of the neonate. The challenge seems to be increasing with each passing day due the escalating multidrugresistant organisms [9]. In practice, ampicillin or amoxicillin in combination with an aminoglycoside (amikacin or gentamicin) is the most common antibiotic regimen for neonatal sepsis.

The use of many classes of antibiotics clearly demonstrated a possible resistance of bacteria involved in sepsis. In fact, the use of broad spectrum antibiotics in the present case may have resulted in antibiotic selection pressure on the bowel inhabitants resulting in increased survival and overgrowth of the resistant population. In case of severe infection due to multidrug-resistant members of the Enterobacteriaceae, including those with extended-spectrum $\beta$-lactamases (ESBLs) or AmpCs, carbapenems and quinolones are used as the last resort for treatment [12]. The choice of antibiotics should also be based on susceptibility reports, type of infection being treated, the severity of the infection and clinical response to the regimen chosen.

\section{CONCLUSION}

The empiric long term antibiotic treatment and the high number of late onset sepsis with often multiresistant Enterobacteriaceae might be causal and urges for a change in general antibiotic prophylaxis/treatment in newborns admitted to the NICU. As described in this case report, the findings demonstrate the need for strict enforcement of infection control practices, whereas an early microbiology screening plays a 
pivotal role in early detection and reporting of antibiotic resistance. In addition, close liaison between the clinicians and the microbiologists will facilitate a significant reduction in morbidity and mortality in NICU. The emergence and spread of these pathogens can be significantly curtailed if appropriate infection control procedures, strict enforcement of antibiotic policies and screening programs are implemented immediately.

\section{ACKNOWLEDGEMENT}

We thank the staff members of the Hospital's NICU for their support in this study.

\section{REFERENCES}

1. Haque KM. Neonatal sepsis in very low birth weight preterm infants: part1: review of Pathophysiology. J. Med. Sci. 2010; 3:1-10.

2. Hornik $C P$, Fort $P$, Clark $R H$, Watt $K$, Benjamin $D K J r$, Smith PB, Manzoni $P$, Jacqz-Aigrain E, Kaguelidou $F$, Cohen-Wolkowiez M. Early and late onset sepsis in very-low-birth-weight infants from a large group of neonatal intensive care units. Early Hum. Dev. 2012; 88: S69-S74.

3. Mclntire, DD, Leveno, KJ. Neonatal Mortality and Morbidity Rates in Late Preterm Births Compared With Births at Term. Obstet. Gynecol. 2008; 111: 35 41.

4. Bakhuizen SE, Haan TR, Teune MJ, Van WassenaerLeemhuis AG, Van der Heyden JL, Van der Ham $D L$, Mol BWJ. Meta-analysis shows that infants who have suffered neonatal sepsis face an increased risk of mortality and severe complications. Acta Paediatr. 2014; 103: 1211-1218.

5. Stoll BJ, Hansen NI, Sánchez PJ, Faix RG, Poindexter $B B$, Van Meurs KP, Bizzarro MJ, Goldberg RN, Frantz ID 3rd, Hale EC et al. Eunice Kennedy Shriver National Institute of Child Health and Human
Development Neonatal Research Network: Early onset neonatal sepsis: the burden of group $B$ Streptococcal and $E$. coli disease continues. Pediatrics 2011; 27:817-826.

6. Kangozhinova K, Abentayeva B, Repa A, Baltabayeva A, Erwa $W$, Stauffer $F$. Culture proven newborn sepsis with a special emphasis on late onset sepsis caused by Enterobacteriaceae in a level III neonatal care unit in Astana, Kazakhstan. Wien Klin.Wochenschr. 2013; 125: 611-615.

7. Zaidi AK, Thaver I, Ali SA, Khan TA. Pathogens, associated with sepsis in newborns and young infants in developing countries. Pediatr. Infect. Dis. J. 2009; 28:10-18.

8. Vergnano $S$, Menson E, Kennea $N$, Embleton $N$, Russell $A B$, Watts $T$, Robinson MJ, Collinson A, Heath PT. Neonatal infections in England: the NeonIN surveillance network. Arch. Dis. Child Fetal Neonatal 2011; 96:F9-F14.

9. Leroux $S$, Zhao W, Bétrémieux $P$, Pladys $P$, Saliba $E$, Jacqz-Aigrain E: Therapeutic guidelines for prescribing antibiotics in neonates should be evidence-based: a French national survey. Arch. Dis. Child DOI: 10.1136/archdischild-2014-306873.

10. Härtel C, Faust K, Avenarius S, Bohnhorst B, Emeis M, Gebauer C, Groneck P, Heitmann F, Hoehn T, Hubert $M$ et al. Epidemic microclusters of bloodculture proven sepsis in very-low-birth weight infants: experience of the German Neonatal Network. PLoS One. 2012;7: e38304.

11. Shantala GB, Nagarathnamma T, Pooja DR, Harsha TR, Karthik R. Neonatal Septicaemia Caused by Vancomycin Resistant Enterococcus Faecium-A Case Report. J.Clin.Diagn. Res. 2014; 8:DD03-DD04.

12. Greenwood C, Morrow AL, Lagomarcino AJ, Altaye M, Taft DH, Yu Z, Newburg DS, Ward DV, Schibler KR. Early empiric antibiotic use in preterm infants is associated with lower bacterial diversity and higher relative abundance of Enterobacter. J.Pediatr. 2014; 165:23-29. 